\title{
Tiene género la creatividad? Obstáculos a la excelencia en mujeres
}

\author{
Does creativity have a gender? Obstacles \\ to excellence in women
}

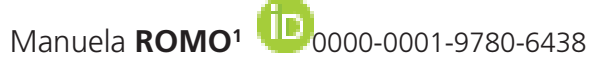

\section{Resumen}

Es un reto social para la cultura de comienzo del milenio conseguir cuanto antes la presencia, en condiciones de igualdad, de mujeres en actividades de producción creativa. Sin embargo, hasta ahora han sido pocos los trabajos que han abordado la cuestión de la reducida presencia de mujeres en actividades relacionadas con la llamada "big-creativity". En el artículo se analizan los condicionantes sociales que dificultan a las mujeres alcanzar el nivel de excelencia en el desarrollo de sus carreras creativas. Desde el constructo "locus de la creatividad" de Csikszentmihalyi, se argumenta sobre las razones de la dificultad de las mujeres para estar en ese "locus" por la dificultad de acceder a los campos o disciplinas y por la ausencia de reconocimiento de sus aportaciones por parte de los ámbitos, es decir, los responsables de admitir en los campos los nuevas aportaciones, al estar ausentes generalmente las mujeres de los mismos. Se analizan también las formas que toma la discriminación como "el techo de cristal o el efecto tijera". Finalmente se argumenta sobre las dificultades de alcanzar la excelencia debido también a la incorporación que las propias mujeres hacen de los prejuicios que las limitan desde la infancia donde comienza la socialización diferencial entre niños y niñas.

Palabras clave: Creatividad; Csikszentmihalyi; Identidad de género; Techo de cristal.

\begin{abstract}
It is a social challenge for the culture of the beginning of the millennium to get as soon as possible the presence, under conditions of equality, of women in activities of creative production. However, until now there have been few works that have addressed the issue of the reduced presence of women in activities related to the so-called "big-creativity". The article analyzes the social constraints that make it difficult for women to reach the level of excellence in the development of their creative careers. From the construct "locus of creativity" of Csikszentmihalyi, it is argued about the reasons for the difficulty of women to be in that "locus" due to the difficulty of accessing domains or disciplines and the lack of recognition of their contributions by the fields, that is, those people responsible for admitting the new contributions in
\end{abstract}

${ }^{1}$ Universidad Autonoma de Madrid, Facultad de Psicología. Ciudad Universitaria de Cantoblanco, 28049, Madrid, España. Dirección de Correspondencia/Correspondence to: M. ROMO.E-mail:<manuela.romo@uam.es>.

$\boldsymbol{v} \boldsymbol{v}$

Como citar este artículo/How to cite this article

Romo, M. (2018). Tiene género la creatividad? Obstáculos a la excelencia en mujeres. Estudos de Psicologia (Campinas), 35(3), $247-258$. http://dx.doi.org/10.1590/1982-02752018000300003 
the domain, since women are usually absent from them. The forms taken by discrimination such as the "glass roof" or the "scissors effect" are also analyzed. Finally, it is argued about the difficulties of achieving excellence due also to the incorporation that women themselves make of the prejudices that limit them from childhood, where the differential socialization between boys and girls begins.

Keywords: Creativity; Csikszentmihalyi; Gender identity; Glass ceiling.

En nuestra era del cambio, la globalización y la información, la creatividad se convierte en un desafío para conformar una ciudadanía de mente abierta, capaz de admitir lo nuevo pero, a la vez, contribuir a la novedad y al cambio. Todas las manifestaciones de la cultura, incluyendo el avance científico y tecnológico se fundamentan en las contribuciones de mentes creativas, capaces de aportar novedad y valor a cada uno de los ámbitos. El progreso económico se vincula a la generación de información, a la innovación y a la creatividad. Así mismo, el desarrollo social se fundamenta en gran medida en las características mencionadas. Como he dicho en otras ocasiones es la creatividad humana la responsable de todo el desarrollo de nuestra civilización, la que nos ha llevado desde las cavernas hasta Saturno (Romo, 2009).

En este contexto constituye un reto social de primera magnitud la presencia de mujeres, en condiciones de igualdad, en actividades de producción creativa en campos como la investigación científica y tecnológica, la arquitectura, la filosofía, las humanidades, las artes plásticas, la música, la danza, el cine, la literatura, la comunicación, el compromiso social o las organizaciones. Sin embargo, las mujeres han estado durante toda la historia, y así continúan en la actualidad, relegadas en sus oportunidades de contribuir a hacer aportaciones a todos los campos de la cultura, casi sin excepción, y de contribuir a forjar esas unidades de información cultural: los memes responsables de la evolución del homo sapiens. Igual que los genes, de acuerdo con Dawkins (2000), aquellas casos que han soportado las presiones de una selección cultural - en este caso - se trasmiten de generación en generación, permanecen enriqueciendo el acervo cultural sean ruedas, bombillas, teléfonos móviles, naves espaciales, quijotes, novenas sinfonías, pirámides que ha sido responsable del avance científico, social, artístico, literario, tecnológico... es el resultado de la obra creativa humana (Csikszentmihalyi, 1998).

Aparte de otras razones de naturaleza antropológica, social o ética... Ante el reto de una sociedad globalizada y en constante cambio no podemos permitirnos prescindir de las contribuciones al desarrollo de la cultura que puede hacer la mitad de la humanidad.

\section{Sobre la excelencia: "big and little creativity"}

Sin embargo, aunque la creatividad es una dimensión universal de la naturaleza humana, existen muchas formas y niveles. La creatividad de los memes no es la misma que se desarrolla en la vida diaria cuando nos encontramos con problemas nuevos en la economía doméstica, en la educación de los hijos, en las actividades de ocio, al innovar en la cocina o en las relaciones laborales; problemas que debemos formular y resolver de forma original y útil.

Los autores han dado denominaciones diversas para diferenciar la creatividad de los memes, a veces de autor desconocido - quien inventó la rueda? quien hizo las pinturas rupestres de la cueva de Altamira? - de la creatividad de la vida diaria. Se diferencia entre Creatividad con mayúscula y creatividad con minúscula, gran y pequeña creatividad o creatividad histórica y creatividad personal (Tabla 1).

Por mi parte, elijo los términos de Boden (1994) que diferencia entre la creatividad de aquellos productos que han pasado a la historia y la creatividad personal de la que todos estamos dotados en mayor o menor medida, enfatizando que no hay diferencias cualitativas desde el punto de vista psicológico. Efectivamente, los genios 


\begin{tabular}{ll}
\hline Creatividad de los genios (eminent creativity) & Creatividad de la gente corriente (everyday creativity) \\
\hline Big-C & Little-C \\
Simonton (1988); Csikszentmihalyi (1998) & Simonton (1988); Csikszentmihalyi (1998) \\
Baer \& Kaufman (2008) & Baer \& Kaufman (2008) \\
Creatividad Historica & Creatividad Personal \\
H-creatividad & P-creatividad \\
Boden (1994) & Boden (1994) \\
\hline
\end{tabular}

no nacieron como tales, fueron P-creativos antes del reconocimiento y muchas de sus obras no se pueden considerar memes. De las miles de obras de Picasso, muchas de ellas no son reconocidas por los expertos como obras maestras, por ejemplo.

Entender la $\mathrm{P}$ nos ayuda a entender la $\mathrm{H}$ y viceversa. Pero, Qué convierte entonces a la $\mathrm{P}$ en $\mathrm{H}$-creatividad?

Hay una serie de requisitos sin los cuales no puede aparecer la $\mathrm{H}$-creatividad y que, como he dicho, no suponen diferencias cualitativas con las personas que no han alcanzado el nivel de la excelencia.

1) Una forma de pensar.

Por mi parte, he definido creatividad de forma operativa, es decir en términos de un producto objetivo, como defendían los positivistas: Creatividad es una forma de pensar cuyo resultado son "cosas" que tienen a la vez novedad y valor (Romo, 1997). Pero el producto, nuevo y valioso, vienen determinado por un proceso sin el cual no se alcanzan esas características. Es una definición que está en la línea de otras muchas y sintetiza sus contenidos, por ejemplo: Gardner (1995, p.53) "El individuo creativo es una persona que resuelve problemas con regularidad, elabora productos o define cuestiones nuevas en un campo de un modo que al principio es considerado nuevo, pero que al final llega a ser aceptado en un contexto cultural concreto". Para Boden "Creatividad es la habilidad para dar con ideas o artefactos que son nuevos, sorprendentes y valiosos" (Boden, 1994, p.52).

No es objeto aquí de entrar en detalle en el asunto de la definición y el análisis de los procesos mentales de la creación (Romo 1997). Sintetizando brevemente afirmamos que aplicar sistemáticamente los procesos propios del pensamiento creador en el área de dedicación en una disciplina significa: buscar y encontrar problemas, formularlos y resolverlos de una forma original y útil; en ese proceso es fundamental el uso de estrategias como la flexibilidad mental para ensayar enfoques diversos ("pensar fuera de la caja") o conectar ideas alejadas entre sí en una eficaz utilización del pensamiento analógico.

2) Unas habilidades de infraestructura, como he denominado a una buena dotación de la inteligencia propia del dominio (Romo, 1997).

La inteligencia de dominio interactúa con las habilidades del pensamiento creador mencionadas para alcanzar la idea creativa. Gardner (1995) en Mentes Creativas ha analizado en profundidad en sus 7 estudios de caso, como tiene lugar esa síntesis mágica para alcanzar la genialidad en Picasso, Stravinsky, Elliot, Graham, Einstein, Freud o Ghandi.

Efectivamente, tenemos que hablar de una conexión muy fuerte con la inteligencia, de un desarrollo extraordinario de ésta para alcanzar el nivel del genio, pero no en la forma en que se planteaba por los años 60, vinculada al Cociente Intelectual (C.I.) en diversos trabajos (Getzels \& Jackson, 1962; Torrance, 1970; Wallach \& Kogan, 1965), sino en los términos de las inteligencias de dominio que Gardner nos ha descrito en su teoría de las inteligencias múltiples (Gardner, 1993).

3) Dominio de toda la información relevante en el dominio. 
Efectivamente, aquí no opera la ciencia infusa. Es necesario haberse formado exhaustivamente en la disciplina, conocer sus entresijos. Para romper un paradigma hay que dominarlo como Kuhn estableció al hablar de las revoluciones científicas (Kuhn, 1962). Solo así se pueden conocer sus limitaciones, plantearse preguntas y encontrar respuestas que lleven a hacer aportaciones relevantes al campo. Pero esta competencia en el campo requiere mucho tiempo; igualmente el desarrollo de la interacción entre ese pensamiento creativo y las habilidades para el campo, lo cual nos lleva al siguiente presupuesto necesario para que aparezca la h-creatividad.

4) Una enorme dedicación al trabajo. Lo que empíricamente se constata en la regla de los 10 años.

Diez años o, lo que es lo mismo, 20.000 horas de trabajo sostenido. Este tiempo es el promedio que se necesita para realizar una obra maestra después de comenzar la inmersión decidida en una disciplina. Regla que enuncio Hayes (1981) hace mucho tiempo en su estudio con músicos y que otros autores después han constatado en estudios de caso. En el libro de Gardner (1995) se verifica la regla en sus 7 creadores de la era moderna. Obviamente la regla define un promedio y las 20.000 horas nadie las ha contado pero es una bonita cifra redonda que aparece cuando consideramos que la profunda dedicación a su actividad de los h-creativos puede suponer 2000 horas de media al año de su trabajo.

5) Una enorme motivación intrínseca hacia el trabajo.

"Por amor al arte", dice el refrán español cuando alguien se ve impelido a realizar una dedicación sin otro fin que la actividad en sí misma, como hace el artista.

En toda la literatura sobre Psicología de la Creatividad aparece este rasgo, sin discusión, como inherente a la conducta creativa: la motivación para ocuparse en una actividad porque es intrínsecamente interesante, agradable o satisfactoria.

Amabile (1983, 1988, 1996), pionera

250 y continuadora de la investigación sobre las conexiones entre motivación intrínseca y creatividad, define así el Principio de la Motivación Intrínseca para la creatividad: La gente será más creativa cuando se sienta motivada primeramente por el interés, placer, satisfacción y desafío del trabajo por sí mismo y no por presiones externas (Amabile, 1988).

Esas presiones externas pueden tener tanto una valencia negativa: competencia, obligación, como positiva: dinero, premios o reconocimiento social. Durante el trabajo creativo quedan excluidas como motivaciones y lo único que cuenta es la propia tarea.

6) Estilos cognitivos y rasgos de personalidad favorecedores.

Las siguientes características se presentan conjuntamente en las personas más creativas (Romo, 2008). Perseverancia: significa mantener el esfuerzo ente los obstáculos que pueden ser externos pero también limitaciones personales que la persona aprende a superar; recordemos, por ejemplo, el caso de Stephen Hawking. Asumir riesgos: se minimiza el temor al fracaso y se afrontan riesgos ante el posible rechazo a la aportación revolucionaria en un campo. Tolerancia a la ambigüedad: resistencia a dominar la incertidumbre, gusto por la complejidad; está muy presente en los creadores, hombres o mujeres, el rasgo de androginia psicológica como una forma de conjugar masculinidad y feminidad en la personalidad creativa. Independencia: en la forma del estilo cognitivo independencia de campo. En el pensamiento se relaciona con la flexibilidad mental: no dejarse llevar, buscar enfoques nuevos y en la conducta social significa formas diversas desde autonomía hasta marginalidad; Gardner en sus 7 estudios de caso nos habla de la marginalidad del creador. Apertura a la experiencia que puede ser interna en la forma de asumir los conflictos internos, cosa muy presente en la literatura, la música o las artes o externa: personas abiertas a la novedad, dispuestas a experimentar, a dejarse sorprender. En el modelo Big Five este es el rasgo más definidor de la personalidad creativa (Dingman, 1990). "Yo no tengo dotes especiales, solo soy apasionadamente curioso" dijo Einstein. Autoconfianza: necesaria para todo lo anterior. Confianza que anima a 
perseverar, afrontar el rechazo, a probar,... Sin un buen autoconcepto la creatividad no es posible.

7) En definitiva, podemos hablar de un estilo de vida.

Para las personas h-creativas la presencia de estas características psicológicas es constante configurando un estilo de vida.

Respecto de las 7 características de la $\mathrm{H}$-creatividad definidas, hay que enfatizar que no existen diferencias entre hombres y mujeres. $\mathrm{Ni}$ en los estudios de caso (Gardner, 1993), Gruber y Wallace (1989) ni en los historiometricos (Simonton, 1988), ni en trabajos cualitativos como el de Csikszentmihaly (1998) con 91 personas destacadas contemporáneas, se constata ninguna diferencia. Tampoco hay diferencias en los estudios psicométricos de diversos autores como Mackinnon (1975); Barron (1972); Getzels y Csikszentmihalyi (1976); Helson (1983, 1999); Feist (1998), Baer y Kaufman (2008)... con personas $\mathrm{H}$ y P-creativas de ambos sexos tanto con artistas, científicos, escritores, emprendedores, Entonces.... Donde están las mujeres eminentes? Por qué nos cuesta tanto encontrarlas?

Para acercarnos a la respuesta debemos considerar que todos estos son requisitos necesarios pero no suficientes. Todos tenemos noticia de mucha gente cuyo estilo de vida es la creatividad pintando, escribiendo, investigando, trabajando por los demás, dirigiendo una pequeña empresa,.... Y no aparecen en la portada de la revista Time ni han sido nominados para el premio Princesa de Asturias,.. Para llegar al Olimpo de la creatividad histórica hacen falta además otros factores que no son de naturaleza psicológica sino social. Estos factores sociales que vamos a considerar ahora constituyen la clave para entender la limitada presencia de las mujeres en los lugares destacados de los artífices del avance de las disciplinas.

Para argumentar en esta explicación de naturaleza social debemos situarnos en un modelo complejo que contempla la creatividad como una dimensión que se encuentra más allá de la mente de una persona. Debemos habar, en términos de Csikszentmihalyi, del locus de la creatividad.

\section{El locus de la creatividad y las diferencias de género}

No es suficiente con un estilo de vida, hace falta que las aportaciones de esa persona pasen a formar parte de una disciplina y que la sociedad las reconozca como originales y valiosas. Aquí está la clave para entender las dificultades de las mujeres para dar el salto de la $\mathrm{P}$ a la $\mathrm{H}$-creatividad. La explicación es de carácter histórico y sociológico y tienen que ver con el "locus de la creatividad", concepto acuñado por Csikszentmihalyi. Como dice el mismo autor: "La creatividad no se produce dentro de la cabeza de las personas, sino en la interacción entre los pensamientos de una persona y un contexto sociocultural" (Csikszentmihalyi, 1998, p.74).

Ya en el año 1988, Csikszentmihalyi planteaba la provocativa pregunta: Donde está la creatividad? Para él, ahí está la clave, más que en responder: qué es la creatividad? La creatividad no solo está en la mente de una persona, debemos considerar a la persona como uno de los 3 sistemas que constituyen su famoso triangulo: persona, campo y ámbito.

Para alcanzar el nivel de la excelencia no basta con una persona con las cualidades que he descrito anteriormente y que lanza un producto a una disciplina o campo de la cultura; es necesario que ese campo incorpore esa obra, que pase a formar parte de la disciplina de manera definitiva y, para que eso suceda, hace falta que los guardianes del campo, los que abren las puertas del campo, como los define Csikszentmihalyi: los expertos, en una palabra, con sus herramientas de conocimiento y experiencia - no arbitrariamente - decidan que merece la pena que ese producto se incorpore a la disciplina y la modifique de manera permanente.

La interrelación entre estos 3 sistemas determina conjuntamente la aparición de la idea, acción o producto creativo. El individuo utiliza información de la cultura a través de su disciplina y la transforma. Si el cambio es considerado valioso por el ámbito se aceptará y la persona pasa a convertirse en h-creativa.

Volviendo al tema del género, lo que sucede sencillamente es que las mujeres tienen 
más problemas para encontrarse en ese locus de la creatividad por su dificultad para acceder al campo y al ámbito. En la mayoría de los campos hay dificultades de acceso para las mujeres y cuando esto sucede, la falta de reconocimiento por parte de los ámbitos, determina la limitada presencia de mujeres en ese locus de la creatividad; dicho de otra manera, explica la escasez de mujeres eminentes. Vamos a ver cómo se han materializado estas dificultades, cuáles son los obstáculos que las mujeres y sólo las mujeres han tenido y tienen para alcanzar la excelencia, pero antes trataré sobre otra forma sutil en que la cultura margina a las mujeres de ese locus de la creatividad. Me refiero a la gran ausencia de estudios sobre la creatividad femenina.

\section{Escasez de estudios sobre mujeres creativas}

La investigación pionera de Barron (1972) sobre diferencias de género en creatividad artística en 1972 fue realizada con estudiantes del Instituto de Arte de San Francisco y de la escuela de diseño de Rode Island. Para ello utilizó el Inventario Multifásico de la Personalidad de Minnesota (M.M.P.I.), observando que ambos géneros mostraban pensamiento independiente, carácter inconformista y poco convencional. Sin embargo las mujeres eran más flexibles, abiertas, enérgicas y destacaban por su sensibilidad a los detalles. También observó que había diferencias significativas en el autoconcepto a favor de los hombres, aunque este aspecto no influía en la calidad de los trabajos de las mujeres, que eran tan valiosos como los de sus compañeros. La diferencia más reseñable relacionada con los diferentes roles sociales atribuido a cada género tenía que ver con la intensidad del compromiso que tienen los jóvenes artistas ante su carrera. Los hombres decían que su trabajo era su vida, una necesidad y su principal razón para vivir; las mujeres sin embargo, daban la misma importancia a su profesión que al cuidado de la familia (Barron, 1972).

El estudio ya clásico de Getzels y 252 Czikszentmihalyi (1976), que sirvió para definir su modelo de "problem finding" sobre el pensamiento creativo, se hizo con 321 estudiantes, 152 mujeres y 169 hombres, que habían estudiado en la School of Arts de Chicago. Comparando entre sí los dos grupos, encontraron que aunque mujeres y hombres eran muy similares en rasgos de personalidad, había algunas diferencias de género: las mujeres artistas mostraron más valores masculinos que sus contemporáneas no artistas y los hombres tenían a su vez más intereses y valores femeninos que los varones no artistas. Este resultado es coherente con el modelo de personalidad paradójica que postula Csikszentmihalyi (1998) y que incluye entre sus 10 pares de rasgos opuestos que aparecen simultáneamente en las personas más creativas, el de masculinidad-feminidad: los artistas -hombres y mujeres- son más andróginos, como hemos dicho en el epígrafe anterior, tienden a estar en el medio de ese continuo. También es coherente esto con la presencia de un estilo cognitivo de tolerancia a la ambigüedad como aparece sistemáticamente en los estudios sobre la personalidad creativa (Romo, 2008; Sawyer, 2012; Sternberg \& Lubart, 1997).

Estos 2 estudios clásicos de carácter diferencial se realizaron con muestras de ambos sexos pero las investigaciones con mujeres exclusivamente son escasas y relativamente recientes. Vamos a reseñar aquí las que consideramos más relevantes en consonancia con los propósitos de nuestro trabajo: aproximarnos a las razones de la escasez de mujeres eminentes.

List y Renzulli (1991) examinaron el impacto de la influencia social en el desarrollo de la creatividad en las mujeres artistas. Para ello estudiaron sus experiencias en educación formal, apoyo familiar, el papel que jugaban los mentores y la visión que tenían ellas de su desarrollo en su propio trabajo creativo. Los resultados indicaron que a pesar de una experiencia negativa en la educación formal, el apoyo familiar era muy importante y señalaban los beneficios de haber tenido al menos la influencia de un mentor en sus vidas que les impulsara a crear.

En un estudio llevado a cabo por Reis (1995) con mujeres de talento que eligieron una carrera aparece un dato interesante sobre la influencia parental para el desarrollo de la creatividad: los 
padres normalmente animaban a sus hijas a alcanzar sus metas en la escuela, pero ese apoyo se redujo durante los años de universidad, especialmente en los casos en que los padres tenían la expectativa de que se casasen y formasen su propia familia en un futuro. Algunas de estas mujeres con talento revelaron que fueron animadas a hacer una carrera profesional siempre que ello no fuera un obstáculo para "anteponer a su familia".

En su artículo de revisión Why are they so few, Piirto (1991) llegaba a conclusiones similares sobre las dificultades sociales para aceptar la realización de una vocación creativa por parte de las mujeres fuera del hogar. En el terreno musical, señaló que incluso aquellas que habían trabajado duro habían sido consideradas más como compañeras y musas. Un ejemplo claro fue Alma Mahler, la esposa de Gustav Mahler; Alma fue una importante compositora, sin embargo, se referían a ella como una musa, una inspiración para los hombres creativos de la época; los términos del contrato matrimonial de Alma con Gustav Mahler incluían el abandono de su vocación artística y musical para dedicarse plenamente su matrimonio, según Piirto.

Existen numerosos ejemplos de colaboraciones, incluso autorías intelectuales, de creaciones destacadas que han sido adjudicados injustamente al miembro varón de una pareja. Ada Lovelace supuesta amante de Charles Babagge - trabajo con él en el proyecto de la máquina analítica que sería la primera computadora: hoy día se reconoce a Lovelace como la autora del primer algoritmo destinado a ser procesado por una máquina, es decir, ella fue la primera persona programadora de ordenadores en la historia. Numerosos pseudónimos masculinos han sido utilizados para poder dar a conocer la obra de una mujer; Concepción Arenal, la escritora española, se disfrazaba de hombre para poder acceder a las clases en la universidad.

Otra consecuencia de este destino doméstico para la mujer puede ser, como señala también Helson (1983), que, en condiciones similares de excelencia académica, los profesores universitarios matemáticos hacen ofertas de futuro científico fundamentalmente a los varones. Parece evidente que si los padres y la sociedad, en general, priorizan el rol de esposa, madre e hija ante cualquier vocación creativa fuera del hogar, es normal que se dé una renuncia a la misma, para evitar la disonancia o bien se afronte esa disonancia acarreando en no pocos casos conflictos y sentimientos de culpa (Piirto, 1991).

En su estudio con mujeres matemáticas seleccionadas por la calidad de sus trabajos, Helson (1983), observó que ninguna de ellas estaba en puestos académicos o en instituciones de investigación, sino que trabajaban en casa pese a que su media de edad rondaba los 41 años. El Inventario Psicológico de California $(\mathrm{CPI})$, reveló que las mujeres creativas en matemáticas valoraban su intelecto y su propia independencia y estaban orgullosas de su objetividad y racionalidad. Estas mujeres puntuaban muy alto en flexibilidad y muy bajo en perseguir metas conformistas y comunes; preferían elegir su propio camino a la hora de hacer las cosas. En su tiempo libre las mujeres creativas se decantaban por las actividades intelectuales tales como escuchar música clásica, dar paseos por la naturaleza, ir al teatro y leer. Por otro lado, las mujeres creativas no obtuvieron resultados similares en el Inventario Psicológico de California respecto a los hombres matemáticos creativos: ellos se describían a sí mismos como seguros, con iniciativa, ambición, influencia sobre el medio, equilibrio intelectual y congruencia. En un estudio longitudinal más reciente sobre personalidad creativa en mujeres, Helson (1999) ratifica, en gran medida, los resultados encontrados con las mujeres matemáticas.

En el mundo empresarial hay una sensibilidad creciente hacia el liderazgo femenino y la promoción de la mujer y nos encontramos con trabajos muy recientes, especialmente en el campo del emprendimiento. Seguramente porque es el ámbito donde, desde el punto de vista económico, interesa más potenciar a las mujeres en un periodo de crisis económica generalizada y duradera como el que hemos vivido desde 2008. El emprendimiento se considera un motor de la innovación y el crecimiento. Prestar especial atención a la implicación de la mujer en este ámbito se hace inevitable en tanto 
representa un grupo social de fuerza relevante en lo que a creación de empresas se refiere (Fuentes \& Sanchez, 2010). Dentro de este ámbito, en España, el estudio de Reyes, Pinillos y Soriano (2014), constata que las mujeres emprendedoras tienen mayor aversión al riesgo y se consideran a sí mismas menos innovadoras pero, por otra parte, son más proactivas que los varones en cuanto a responsabilidad, disciplina e independencia, mientras que los hombres tienen mayor iniciativa y capacidad de liderazgo.

Otros estudios hablan del estilo de liderazgo femenino en el mundo empresarial. A pesar de la baja representación en puestos directivos es muy interesante el metaanálisis de Eagly, JohannesenSchmidt y Van Engen (2003) que combinó los resultados de estudios de líderes en el ámbito de los negocios, estudios y otras áreas. Se encontró, que las mujeres utilizaban un estilo de liderazgo que resultaba más efectivo, que el utilizado por los hombres; concretamente, se encontró, que era más probable que las mujeres fuesen líderes transformacionales que generan entornos de trabajo más productivos y satisfactorios. Este tipo de liderazgo se basa en la motivación y en la transmisión de los objetivos de la empresa al empleado y busca motivar al trabajador y propiciar la generación de ideas, estimulando sus habilidades y creatividad; también promueve la confianza entre el trabajador y el equipo buscando el crecimiento y desarrollo a largo plazo, más que unos resultados a corto plazo (Puccio \& Cabra, 2010).

\section{Obstaculos a la eminencia en mujeres}

En este recorrido de las escasas y no siempre sistemáticas investigaciones sobre mujeres y creatividad podemos ir aventurando los posibles motivos por los cuales pocas mujeres se atreven a trascender en su carrera: es obvio que las razones son de naturaleza social y que pueden llegar a convertirse en personales en casos como la aparición de culpabilidad de las mujeres que son creativas e intentan compatibilizar trabajo creativo con vida familiar o la presencia de menor necesidad de logro o ambición, tal como se está constatando en los estudios sobre emprendimiento y género.
El techo de cristal y el efecto tijera son 2 metáforas que explican muy bien la forma en que esos obstáculos se hacen operativos.

El techo de cristal es una forma de sutil discriminación de carácter complejo que dificulta escalar puestos de responsabilidad superior en organizaciones de cualquier naturaleza o alcanzar un reconocimiento, al mismo nivel que el varón en aportaciones a la cultura sean del área que sean. Siempre tenemos que volver al triangulo de Csikszentmihalyi, al locus de la creatividad y a los guardianes de las puertas del campo que deciden quien las puede traspasar y quién no: quien alcanzará el reconocimiento y la eminencia. Hay muchas estadísticas que podríamos traer aquí de cualquier área de la cultura para dar cuenta de lo difícil que resulta romper ese techo. Tan solo hago mención a los premios Nobel como el ejemplo-creo- más significativo: hasta 2017 solo 49 mujeres han sido galardonadas en toda la historia frente a 833 hombres. En el caso de los Nobel en ciencias la discriminación es todavía mayor: desde 1901, el 97\% de los ganadores de premios Nobel de ciencias han sido hombres. Solo 18 mujeres se incluyen en el grupo de 590 nobeles científicos. En este siglo largo, los hombres se han llevado el 99\% de los Nobel de Física, el 98\% de los de Química y el $94 \%$ de los de Medicina. Estos datos resultan más escandalosos si consideramos que entre los aspirantes hay proporciones similares de hombres y mujeres $y$, en especial, si consideramos que esta discriminación sistemática no se contempla como un problema.

Parece que el techo de cristal continúa presente como una manera sutil de discriminación que afecta también a la consecución del rango de eminencia por parte de las mujeres en sus trabajos creativos. Y, en ámbitos como el académico, como hemos visto en el trabajo de Helson con matemáticas, el "efecto tijera" sigue recortando las posibilidades de alcanzar la excelencia al nivel de los pares varones. Un 60\% de personas licenciadas en Europa son mujeres, pero representan poco más del 13\% de los puestos de dirección (Unión Europea: Estrategia Igualdad de Género 2010-2015); 
además, este informe sostiene que las mujeres, a pesar de tener una formación académica mejor que los hombres de su edad, buscan empleo en sectores que están relativamente peor pagados, o bien aceptan empleos a tiempo parcial.

Por último, como un ejemplo muy simbólico, presento el informe de la Unidad de Igualdad de Género de mi Universidad, la Universidad Autónoma de Madrid, donde se comparan los datos sobre presencia de hombres y mujeres desde el nivel de estudiante hasta el de catedrático que es el mayor en la escala académica, comparando datos de 2008 y 2013. Aunque la tijera parece que empieza cerrarse sin embargo, es muy llamativo el efecto: cuando las jóvenes constituyen un $60 \%$ de los estudiantes, sin embargo, solo hay un $20 \%$ de mujeres catedráticas (Figura 1).

Las dificultades para las mujeres comienzan con la discriminación en el acceso a la enseñanza, con la socialización diferencial y, aún superada estas dificultades, con el control por parte de los hombres de la norma por la que un determinado logro se considera o no creativo. Según la metáfora del locus de la creatividad de Csikszentmihalyi (1998) significa esto que aunque últimamente se permita para las mujeres un mayor acceso al campo, sin embargo las aportaciones que hagan al mismo van a tener mayores dificultades en ser reconocidas por los ámbitos, todavía en manos de los hombres en gran mayoría como se ve de forma diáfana en la concesión de los Nobel; en consecuencia, el acceso a la eminencia queda restringido (Baer \& Kaufman, 2008)

\section{Socialización diferencial: el origen de la discriminación}

Lo que señalan sistemáticamente los estudios sobre creatividad femenina tienen que ver con esa socialización diferencial que comienza en la familia y en la escuela. En el nivel más lúdico, la diferenciación clásica entre juguetes típicamente de niños y de niñas ya establece el rol de cuidadora que la persona del género femenino ejercerá durante

Evolución de la presencia de mujeres y hombres

en distintos niveles educativos y categorías docentes.

2008-2009 y 2012-2013

GRÁFICO 8

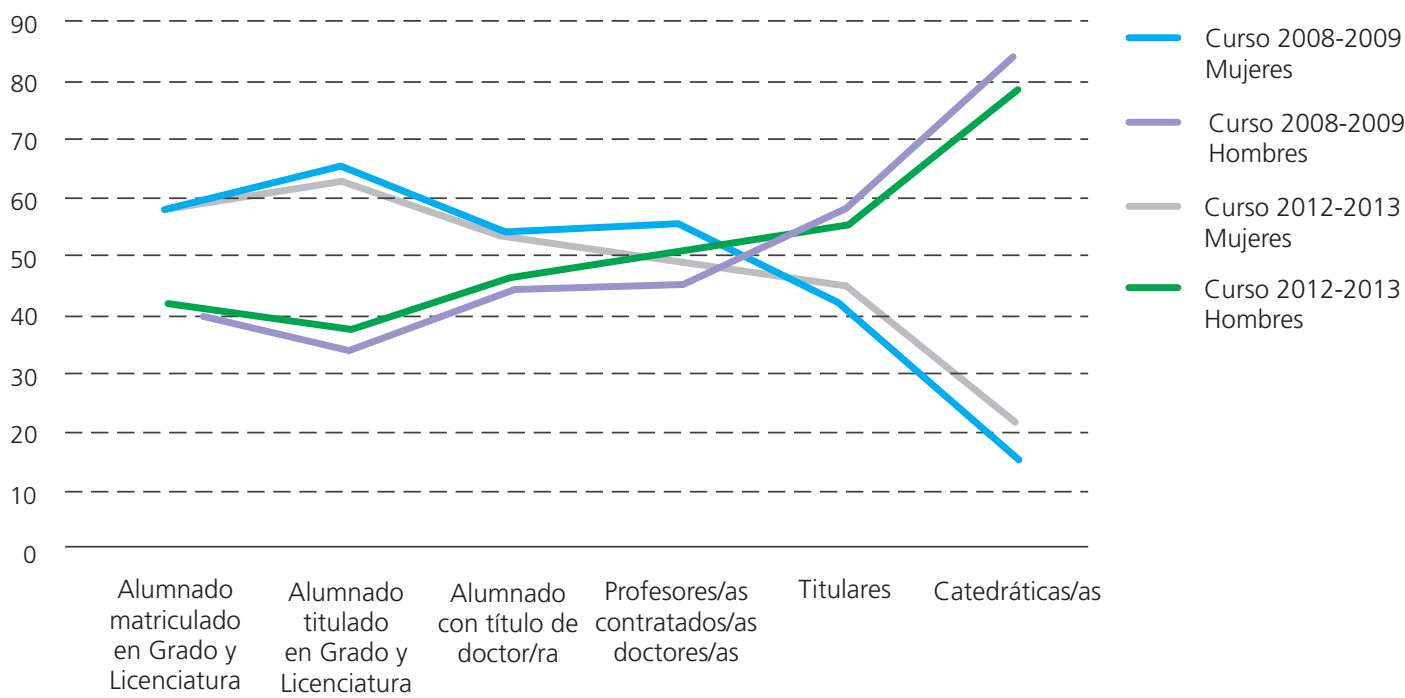

Figura 1. Informe de la Unidad de Igualdad de Género. (Universidad Autónoma de Madrid, 2015) 
su vida. La introyección de roles diferentes resultará muy difícil de modificar después. (Baer \& Kaufman, 2008; Helson, 1983, 1999; Piirto, 1991; Reis, 1995, 2010).

En una reciente investigación de Bian, Leslie y Cimpian (2017) publicada en la revista Science, estos autores trabajaron en 3 ciudades de USA con 400 niños. Los resultados son enormemente preocupantes porque demuestran que hoy seguimos educando de manera diferencial a los niños y las niñas desde la temprana infancia, en perjuicio de estas, ya que a los 6 años las niñas ya consideran que ser listo es un atributo masculino. En la primera prueba, se leyó a un grupo de 96 niños y niñas de cinco, seis y siete años de edad, una historia sobre una persona muy inteligente, $y$ se les pidió que adivinaran el sexo de la persona. Luego se les presentó una serie de imágenes que mostraban pares de adultos, algunos con personas del mismo sexo, otros de sexo opuesto y se les pidió que escogieran los que pensaban que era muy inteligentes. Finalmente, se les pidió que combinaran ciertos rasgos, tales como "ser listo", con imágenes de hombres y mujer. Los resultados del estudio muestran que los niños y niñas de 5 años son capaces de asociar brillantez con su propio género. En cambio a los 6 años las niñas ya han incorporado los estereotipos de género: piensan que brilliance or giftedness es algo más común en los hombres que en las mujeres.

Consecuencia de esta asunción de roles es que el trabajo de las mujeres a menudo difiere del idílico escenario de la actividad creativa. Muchas se enfrentan al dilema de elegir entre su dedicación creativa o atender a las necesidades impuestas por una sociedad que reclama de ellas dedicarse a las obligaciones familiares; A la atención, casi en exclusiva de niños y mayores; desafiando, por otra parte, el estigma de estar menos dotadas para las actividades que requieren innovación y creatividad.

\section{Creadores y creadoras: diferencias en la personalidad como consecuencia de estos obstáculos}

Como consecuencia de la presión de 256 todos los obstáculos que hemos mencionado, nos encontramos con que surge otro techo: el Techo de Cemento. Esta imagen se refiere a las dificultades autoimpuestas por las propias mujeres para crecer profesionalmente, debido a la falta de referentes, la maternidad, la vida personal, una mayor autocrítica; el techo de cemento lo ponen las mujeres que deciden no querer promocionar por el alto coste personal y familiar que va a tener su nueva situación profesional. Esto puede explicar por qué muchas iniciativas de empoderamiento de la mujer han fracasado.

Parece muy difícil eludir el compromiso social como cuidadoras que las mujeres tienen adjudicado. Esto es compatible con algunos datos de la vida familiar que observamos en muchas mujeres creativas tanto en nuestra investigación como en las precedentes:

- Apoyo por parte del compañero ("detrás de una gran mujer hay un gran hombre"!). La misma circunstancia que encontró Csikszentmihalyi en sus 91 creadores, hombres o mujeres.

- Interrupción de su trabajo durante unos años durante la crianza casi en exclusiva de los hijos con las consecuencias negativas para su carrera. Otras han tenido los hijos pronto y han tenido carreras más retardadas. Y otra, finalmente han renunciado a la maternidad.

Finalmente además de estás particularidades de la vida familiar, constatamos en términos psicológicos -de acuerdo con la literatura consultadaque esos obstáculos determinan que las escasas mujeres que han decidido dedicarse plenamente a un trabajo creativo y alcanzar metas de excelencia presentan diferencias claras con sus pares masculinos que afectan a la personalidad y actitudes (Tabla 2).

Para concluir quiero referirme a las conclusiones del informe realizado por el World Economic Forum en 2014 respecto a las diferencias de género a nivel internacional, que tiene en cuenta aspectos básicos como educación, salud, economía y política. Dicho informe estima que aún se tardarán otros 81 años en acabar con las diferencias de género! Casi un siglo resulta inaceptable en una era de globalización y continuo cambio.

A nivel internacional, se necesita una transformación en las condiciones sociales y 
Características generales en la personalidad creativa, presentes en ambos sexos y características exclusivas de las mujeres

\begin{tabular}{ll}
\hline Características favorables a la creatividad en mujeres-hombres & Constricciones a la creatividad en mujeres \\
\hline - Motivación intrínseca & - Autocrítica \\
- Apertura & - Mayor sensibilidad al feedback interpersonal \\
- Perseverancia & - Autoexigencia \\
- Asunción de riesgos & - Aislamiento social \\
- Autoconfianza & - Culpa \\
- Independencia & \\
- Tolerancia a la ambigüedad & \\
\hline
\end{tabular}

económicas que causan esta desigualdad y esto supone un cambio en la percepción de las posibilidades laborales y las capacidades creativas de las mujeres. Para ello se necesita formar en creatividad e igualdad a niños y jóvenes de ambos sexos. Una educación que contrarreste todos los sesgos que, tal como hemos ido viendo, desde el principio de la vida se van interiorizando por los 2 géneros en perjuicio para las niñas, jóvenes y adultas. La educación es un arma cargada de futuro.

\section{Referencias}

Amabile, T. M. (1983). The social psychology of creativity. New York: Springer-Verlag.

Amabile, T. M. (1988). The conditions of creativity. In R. Sternberg (Ed.), The nature of creativity. Cambridge: Cambridge University Press.

Amabile, T. M. (1996). Creativity in context: Update to "The Social Psychology of Creativity". Boulder: Westview Press.

Baer, J., \& Kaufman, J. (2008). Gender differences in creativity. Creativity Research Journal, 42(2), 75-105.

Barron, F. (1972). Artists in the making. San Francisco: Seminar Press.

Bian, L., Leslie, S., \& Cimpian, A. (2017). Gender stereotypes about intellectual ability emerge early and influence children's interests. Science, 355(6323), 389-391.

Boden, M. (1994). La mente creativa: mitos y mecanismos. Barcelona: Gedisa.

Csikszentmihalyi, M. (1988). Society, culture and person: A systems view of creativity. In R. Sternberg (Ed.), The nature of creativity. Cambridge: Cambridge University Press.
Csikszentmihalyi, M. (1998). Creatividad: el fluir y la psicología del descubrimiento y la invención. Barcelona: Paidós.

Dawkins, R. (2000). El gen egoísta. Barcelona: Salvat.

Dingman, J. M. (1990). Personality structure: The emergence of the five-factor model. Annual Review of Psychology, 41, 417-440.

Eagly, A. H., Johannesen-Schmidt, M. C., \& Van Engen, M. L. (2003). Transformational, transactional, and laissez-faire leadership styles: A meta-analysis comparing women and men. Psychological Bulletin, 129(4), 569-591.

Feist, G. J. (1998). A meta-analysis of personality in scientific and artistic creativity. Personality and Social Psychology Review, 2(4), 290-309.

Fuentes, F. J., \& Sánchez, S. M. (2010). Análisis del perfil emprendedor: una perspectiva de género. Estudios de Economía Aplicada, 28(3), 1-28.

Gardner, H. (1993). Inteligencias multiples: la teoría en la práctica. Barcelona: Paidos.

Gardner, H. (1995). Mentes creativas: una anatomía de la creatividad. Barcelona: Paidos.

Getzels, J. W., \& Czikszentmihalyi, M. (1976). The creative vision: A study of problem finding in art. New York: Wiley.

Getzels, J. W., \& Jackson, P. E. (1962). Creativity and intelligence. New York: Willey and Sons.

Gruber, H., \& Wallace, D. (Eds.). (1989). Creative people at work. Oxford: Oxford University Press.

Hayes, J. R. (1981). The complete problem solver. Philadelphia: Franklin Institute Press.

Helson, R. (1983). Creative mathematicians. In R. Albert (Ed.), Genius and eminence: The social psychology of creativity and exceptional achievement (pp.311-330). London: Pergamon Press.

Helson, R. (1999). A longitudinal study of creative personality in women. Creativity Research Journal, 12(2), 89-101. 
Kuhn, T. S. (1962). The structure of scientific revolutions. Chicago: University of Chicago Press.

List, K., \& Renzulli, J. (1991). Creative women's developmental patterns thorough age thirty-five. Gifted Education International, 7(3), 114-122.

MacKinnon, D. (1975). IPAR's contribution to the conceptualization and study of creativity. In J. Getzels \& I. A. Taylor (Eds.), Perspectives in creativity. Chicago: Aldine.

Piirto J. (1991). Why are there so few? (Creative women: Visual artists, mathematicians, musicians). Roeper Review, 13(3), 142-147.

Puccio, G. J., \& Cabra, J. F. (2010). Organizational creativity: A systems approach. In J. Kaufman, C. James, \& R. Sternberg (Eds.), The Cambridge Handbook of Creativity (pp.145-173). Cambridge: Cambridge University Press.

Reis, S. M. (1995). Talent ignored, talent diverted: The cultural context underlying giftedness in females. Gifted Child Quarterly, 39(3), 162-170.

Reis, S. M. (2010). Toward a theory of creativity in diverse creative women. Creativity Research Journal, 14(3-4), 305-316.

Reyes, L. E., Pinillos, L. J., \& Soriano, I. (2014). Diferencias de género en la orientación emprendedora. Esic Market Economics and Business Journal, 45(3), 441-460.
Romo, M. (1997). Psicología de la creatividad. Barcelona: Paidos.

Romo, M. (2008). Creatividad en los dominios artístico y científico y sus correlatos educativos. In M. F. Morais \& S. Bahia. Criatividade: conceito, necessidades e intervenção. Braga: Psiquilibrios Edições.

Romo, M. (2009). Pensamiento creador para los tiempos de crisis. Encuentros Multidisciplinares, 11(31), 27-32.

Runco, M. (2007). Creativity: Theories and themes. Boston: Elsevier Academic Press.

Sawyer, R. K. (2012). Explaining creativity: The science of human innovation. Oxford: Oxford University Press.

Simonton, D. K. (1988). Scientific genius: A psychology of science. Cambridge: Cambridge University Press.

Sternberg, R. J., \& Lubart, T. I. (1997). La creatividad en una cultura conformista. Barcelona: Paidos.

Torrance, E. P. (1970). Encouraging creativity in the classroom. Dubuque: William Brown.

Wallach, M. A., \& Kogan, N. (1965). Modes of thinking in young children: A study of the creativity-intelligence distintion. New York: Holt, Rinehart and Winston.

Recibido: Noviembre 8, 2017

Aprobado: Diciembre 15, 2017 